\title{
Critical dynamics and effective exponents of magnets with extended impurities
}

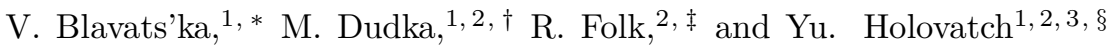 \\ ${ }^{1}$ Institute for Condensed Matter Physics, National Academy of Sciences of Ukraine, UA-79011 Lviv, Ukraine \\ ${ }^{2}$ Institut für Theoretische Physik, Johannes Kepler Universität Linz, A-4040, Linz, Austria \\ ${ }^{3}$ Ivan Franko National University of Lviv, UA-79005 Lviv, Ukraine
}

(Dated: July 8, 2021)

\begin{abstract}
We investigate the asymptotic and effective static and dynamic critical behavior of $(d=3)$ dimensional magnets with quenched extended defects, correlated in $\varepsilon_{d}$ dimensions (which can be considered as the dimensionality of the defects) and randomly distributed in the remaining $d-\varepsilon_{d}$ dimensions. The field-theoretical renormalization group perturbative expansions being evaluated naively do not allow for the reliable numerical data. We apply the Chisholm-Borel resummation technique to restore convergence of the two-loop expansions and report the numerical values of the asymptotic critical exponents for the model A dynamics. We discuss different scenarios for static and dynamic effective critical behavior and give values for corresponding non-universal exponents.
\end{abstract}

PACS numbers: 61.43.-j, 64.60.Ak, 75.10.Hk

\section{INTRODUCTION}

Critical properties of structurally disordered magnets remain a problem of great interest in condensed matter physics, since real magnetic crystals are usually non ideal. A simple and natural case of disorder is implemented via the point-like uncorrelated quenched nonmagnetic impurities and is experimentally realized as substitutional disorder in uniaxial [1] as well as in Heisenberg [2, 3] magnets. Examples are given by substitute alloys $\mathrm{Mn}_{\mathrm{x}} \mathrm{Zn}_{1-\mathrm{x}} \mathrm{F}_{2}, \mathrm{Fe}_{\mathrm{x}} \mathrm{Zn}_{1-\mathrm{x}} \mathrm{F}_{2}$ for the uniaxial (Ising) magnets [4], and by amorphous magnets $\mathrm{Fe}_{90+\mathrm{x}} \mathrm{Zr}_{10-\mathrm{x}}$, $\mathrm{Fe}_{90-\mathrm{y}} \mathrm{M}_{\mathrm{y}} \mathrm{Zr}_{10}(\mathrm{M}=\mathrm{Co}, \mathrm{Mn}, \mathrm{Ni})$ 5, 6], transition-metal based magnetic glasses 7, 8] as well as disordered crystalline materials $\mathrm{Fe}_{100-{ }_{x}} \mathrm{Pt}_{\mathrm{x}}$ 9], $\mathrm{Fe}_{70} \mathrm{Ni}_{30}$ [8] and Euchalcogenide solid solutions [10] for the Heisenberg magnets. The question of a great interest arising here is: does the disorder change critical properties of the systems? The answer is given by the famous Harris criterion 11] It states, that disorder changes the critical exponents, if the critical exponent $\alpha_{p}$ of the pure system is positive: $\alpha_{p}=2-d \nu_{p}>0$. Here, $\nu_{p}$ is the critical exponent governing the divergence of correlation length of the corresponding pure system and $d$ is the space dimension.

On the base of this inequality, one can estimate the marginal value $m_{c}$ for the spin $m$-vector model of magnetic systems, such that for $m>m_{c}$ the critical exponents remain unchanged by point-like defects whereas for $m<m_{c}$ they cross over to new values. The present estimates for $m_{c}$ in three dimensions definitely imply $m_{c}<2$ : $m_{c}=1.942 \pm 0.026[12]$ and $m_{c}=1.912 \pm 0.004$ [13] and thus only the pure Ising model $(m=1)$ is affected by weak point-like uncorrelated disorder at criticality.

\footnotetext{
*viktoria@icmp.lviv.ua

† maxdudka@icmp.lviv.ua

f folk@tphys.uni-linz.ac.at

hol@icmp.lviv.ua
}

But in real magnets one encounters non-idealities of structure, which can not be modelled by simple point-like uncorrelated impurities. Indeed, magnetic crystals often contain defects of a more complex structure: linear dislocations, disclinations, complexes of non-magnetic impurities, embedded in the matrix of the original crystal 14]. Theoretical studies of critical behavior of magnets containing such "extended" (macroscopic) defects have attracted considerable interest 15, 16, 17, 18, 19, 20, 21, 22, 23, 24, 25, 26], however, a systematic experimental analysis still remains to be performed.

One of the possible ways to treat defects that extend through the system being randomly distributed in space but oriented in the same direction is to consider them as quenched $\varepsilon_{d^{-}}$dimensional nonmagnetic impurities of parallel orientation. This was proposed in the work of Dorogovtsev [15]. The case $\varepsilon_{d}=0$ is associated with point-like defects, and extended parallel linear (planar) defects are described by $\varepsilon_{d}=1(2)$. To give an interpretation to the non-integer values of $\varepsilon_{d}$, one may consider patterns of extended defects like aggregation clusters, and treat $\varepsilon_{d}$ as the fractal dimension of these clusters 19. However, relation of analytically continued non-integer Euclidean dimension to the fractal dimension is not straightforward 27.

It was shown [16], that presence of extended impurities leads to a generalized Harris criterion, namely, in this case disorder alters the critical behavior of the pure system, if $\varepsilon_{d}>d-2 / \nu_{p}$. If one considers the pointlike disorder with $\varepsilon_{d}=0$, the generalized Harris criterion turns to ordinary one cited above. Again, this inequality defines for each value of $\varepsilon_{d}$ the critical value $m_{c}$, below which the extended disorder alters the universality class. But now the disorder induced critical behavior of $d=3$ systems holds not only for the Ising magnets $(m=1)$ but for $m>1$ as well, as shown in Fig. 1 (region denoted as "Diluted" in the figure). Therefore, the class of magnets where the new critical behavior may be found is not restricted to the uniaxial ones $(m=1)$, but include easyplane $(m=2)$ and Heisenberg $(m=3)$ systems. Thus 
predicted phenomena of a new universal behavior may be experimentally checked for a wider class of magnets.

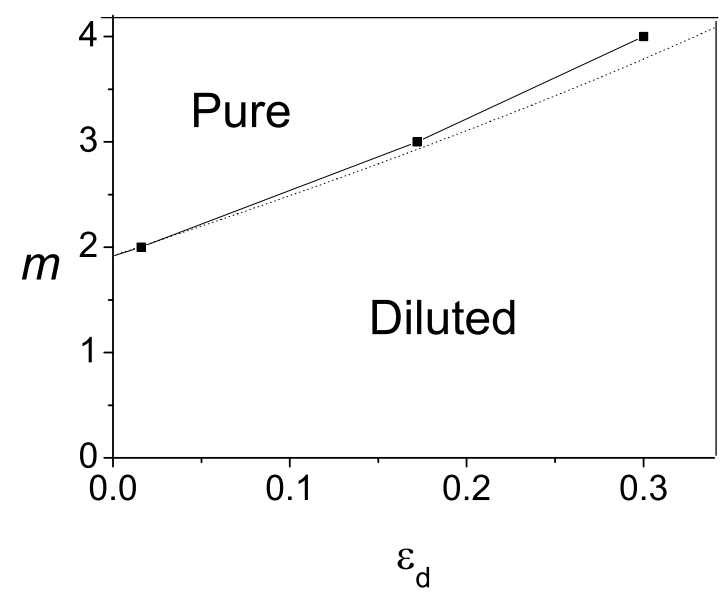

FIG. 1: The phase diagram of $d=3$-dimensional $m$ vector magnets in the presence of $\varepsilon_{d}$-dimensional impurities. Marginal value $m_{c}\left(\varepsilon_{d}\right)$ separates regions of different universality classes. Disorder is relevant in the $\varepsilon_{d}-m$ plane below the $m_{c}$ curve. Filled squares: results of calculations, based on sixloop renormalization group data [28] for the correlation length critical exponent, dotted line: estimate based on resummed $\varepsilon, \varepsilon_{d}$-expansion for $m_{c}$, Eq. (30). Only the physical region $\varepsilon_{d}>0$ is shown. See Section IV for a whole description.

Another interesting feature of systems with parallel extended defects is that due to the spatial anisotropy they are described by two correlation lengths, one perpendicular, $\xi_{\perp}$, and one parallel, $\xi_{\|}$, to the extended impurities direction [15]. As the critical temperature $T_{c}$ is approached, their divergences are characterized by corresponding critical exponents $\nu_{\perp}, \nu_{\|}$:

$$
\xi_{\perp} \sim|t|^{-\nu_{\perp}}, \quad \xi_{\|} \sim|t|^{-\nu_{\|}},
$$

where $t$ is the reduced distance to the critical temperature $t=\left(T-T_{c}\right) / T_{c}$. Anisotropic scaling holds also for the spin-spin pair correlation function at $T_{c}$ and is governed by exponents $\eta_{\perp}$ and $\eta_{\|}$. Whereas the magnetic susceptibility is isotropic, as far as all order parameter components interact with defects in a similar way. However, the dynamic critical behavior is modified, two times of relaxation in directions perpendicular and parallel to the extended impurities $\tau_{\perp}$ and $\tau_{\|}$behave correspondingly as:

$$
\tau_{\perp} \sim \xi_{\perp}^{z_{\perp}}, \quad \tau_{\|} \sim \xi_{\|}^{z_{\|}}
$$

with dynamical exponents $z_{\perp}$ and $z_{\|}$.

Therefore, magnets with quenched extended defects of parallel orientation constitute a large class of systems with a bulk of unusual phenomena worth to be analyzed. Their asymptotic critical behavior was a subject of the field-theoretical renormalization group (RG) analyses [15, 16, 17, 20, 21]. In particular, a double expansion in both $\varepsilon=4-d, \varepsilon_{d}$ was suggested and RG functions were calculated [15] to order $\varepsilon, \varepsilon_{d}$; qualitatively, the crossover to a new universality class in the presence of extended defects was supported. These calculations were extended to the second order in Refs. 16, 18]. However, these divergent RG expansion did not give a reliable numerical estimate for the critical exponents - the goal highly desirable both for experimental and simulational purposes. Numerical estimates for the exponents describing static critical behavior of $d=3$ magnets with parallel extended impurities were obtained only recently [20, 21] by applying special resummation technique to the two-loop RG functions of Refs. 16, 18. Currently, no estimates of similar accuracy exist for the dynamic exponents describing critical slowing down in the magnets under consideration. The theoretical investigation of critical dynamics of system with parallel extended defects was performed in the one-loop approximation [17] for the simple models with non-conserved order parameter (model A) and conserved order parameter (model B). Then the RG study of model A critical dynamics was extended to the twoloop order [18]. But again, the convergence properties of the series obtained did not allow numerical evaluations. Recently, the short-time critical dynamics of the model A was considered in Ref. [22].

The goal of this paper is to apply the state-of-the-art analysis of the divergent RG perturbation theory series to get numerical estimates of the exponents describing model A dynamics of $d=3$ magnets with extended impurities for a wide region of impurity dimension $\varepsilon_{d}$ and for different values of $m$. Complementing existing estimates of the static exponents [20, 21], our results will give comprehensive values for the critical exponents of systems with extended impurities and, in this way, will facilitate their experimental analysis. Moreover, since both in experiments and in Monte Carlo (MC) simulations often the asymptotic region is not reached and effective exponents are observed [29], we will calculate these as well. By these estimates we will predict the possible scenarios of approaching the critical point. These are important to perform corresponding experimental checks, as it was shown recently by theoretical [30] and experimental 31. studies of the $d=3$ disordered Heisenberg magnets with point-like defects.

The setup of the paper is as follows. In the next section we present the model; in section $\amalg$ the renormalization procedure is discussed. In section IV we apply the resummation techniques to analyze the renormalization group functions in two-loop approximation and present the quantitative estimates for the asymptotic dynamical critical exponents. Section $\square$ gives description of possible scenarios for the effective critical behavior and section DI concludes our study. 


\section{THE MODEL}

The starting point is the effective Hamiltonian of the model of an $m$-vector magnet with $\varepsilon_{d}$-dimensional defects, extending throughout the system along the coordinate directions symbolized as $x_{\|}$and randomly distributed in perpendicular directions $x_{\perp}$ [16, 18]:

$$
\begin{aligned}
\mathcal{H}= & \int d^{d} x\left[\frac{1}{2}\left(\mu_{0}^{2}+V(x)\right) \vec{\phi}^{2}(x)+\left(\nabla_{\perp} \vec{\phi}(x)\right)^{2}\right. \\
& \left.+a_{0}\left(\nabla_{\|} \vec{\phi}(x)\right)^{2}+\frac{u_{0}}{4 !}\left(\vec{\phi}^{2}(x)\right)^{2}\right]
\end{aligned}
$$

Here, $\vec{\phi}$ is an $m$-component vector field: $\vec{\phi}=\left\{\phi^{1} \cdots \phi^{m}\right\}$, $\mu_{0}$ and $u_{0}$ are the bare mass and the coupling, $a_{0}$ is the bare anisotropy constant, $\nabla_{\|}$and $\nabla_{\perp}$ denote differentiation in the coordinates $x_{\|}$and $x_{\perp}$ and the impurity potential $V(x)$ is introduced. The probability distribution of defects has zero mean and variance given by:

$$
\langle\langle V(x) V(y)\rangle\rangle=-v_{0} \delta^{d-\varepsilon_{d}}\left(x_{\perp}-y_{\perp}\right) .
$$

Here, $\langle\langle\ldots\rangle\rangle$ denotes the averaging over the distribution of defects, $\left(-v_{0}\right)$ is a positive coupling constant, which is proportional to the concentration of impurities. The constant $a_{0}$ parameterizes the anisotropy, arising in the system due to the presence of extended defects.

We consider critical dynamics of the model (9) for the case of a non-conserved order parameter. For this case the dynamics can be expressed in the Langevin equation form [32]:

$$
\frac{\partial \phi^{i}(x, t)}{\partial t}=-\lambda_{0} \frac{\partial \mathcal{H}}{\partial \phi^{i}(x, t)}+\eta^{i}(x, t), \quad i=1 \ldots m
$$

where $\lambda_{0}$ is the Onsager kinetic coefficient and $\eta^{i}(x, t)$ is the Gaussian random-noise source with zero mean and correlation:

$$
\left\langle\eta^{i}(x, t) \eta^{j}\left(x^{\prime}, t^{\prime}\right)\right\rangle=2 \lambda_{0} \delta\left(x-x^{\prime}\right) \delta\left(t-t^{\prime}\right) \delta_{i j}
$$

The brackets $\langle\ldots\rangle$ mean an average with respect to the thermal noise.

Within the field theory approach it is convenient to use the Bausch-Janssen-Wagner formulation [33] which is given by the Lagrangian:

$$
\mathcal{L}[\tilde{\phi}, \phi]=\int d^{d} x d t \sum_{i} \tilde{\phi}^{i}\left[\frac{\partial \phi^{i}}{\partial t}+\lambda_{0} \frac{\delta \mathcal{H}}{\delta \phi^{i}}-\lambda_{0} \tilde{\phi}^{i}\right]
$$

Here, $\tilde{\phi}^{i}$ are components of an auxiliary response field introduced to average over the thermal noise. Then correlation and response functions are computed with the help of a weight function $W \sim e^{-\mathcal{L}[\tilde{\phi}, \phi]}$.

It is known that studying dynamical properties of disordered systems averaging over random impurities can be applied directly to dynamical weight function $W$. As it is established in Ref. 34], the configurational averaging can be performed avoiding replica trick [35]. However it leads to the same perturbative expansions for the RG functions as those obtained with replica formalism. Therefore both approaches giving equivalent results are possible for dynamics. However the above alternative does not exist when the static critical behavior is analyzed (see e.g. 1, 2]). As far as both static and dynamic criticality is addressed in this paper, we use the replica trick, representing the logarithm of a weight function in the following form:

$$
\ln W=\lim _{n \rightarrow 0} \frac{\left\langle\left\langle W^{n}-1\right\rangle\right\rangle}{n} .
$$

Finally it leads to study of properties of replicated Lagrangian $\mathcal{L}[\tilde{\phi}, \phi][36]$ :

$$
\begin{aligned}
\mathcal{L}[\tilde{\phi}, \phi]= & \sum_{\alpha}\left\{\int d^{d} x d t \sum_{i} \tilde{\phi}_{\alpha}^{i}\left[\frac{\partial \phi_{\alpha}^{i}}{\partial t}+\lambda_{0}\left(\mu_{0}^{2}-\nabla_{\perp}^{2}-a_{0} \nabla_{\|}^{2}\right) \phi_{\alpha}^{i}-\lambda_{0} \tilde{\phi}_{\alpha}^{i}+\sum_{j} \lambda_{0} \frac{u_{0}}{3 !} \phi_{\alpha}^{i} \phi_{\alpha}^{j} \phi_{\alpha}^{j}\right]\right. \\
& \left.+\sum_{i, j, \beta} \lambda_{0}^{2} \frac{v_{0}}{2} \int d^{d} x d^{d} y d t d t^{\prime} \delta\left(x_{\perp}-y_{\perp}\right) \phi_{\alpha}^{i}(x, t) \phi_{\alpha}^{i}(x, t) \phi_{\beta}^{j}\left(y, t^{\prime}\right) \phi_{\beta}^{j}\left(y, t^{\prime}\right)\right\},
\end{aligned}
$$

here, the summation over Greek indices spans from 1 to $n$ denoting the different replicas and the Latin indices go from 1 to $m$ denoting the components of the order parameter. To study behavior of this model (9) in the vicinity of the critical point we apply the minimal subtraction scheme within field-theoretical RG. The description of this approach is given in the next section.

\section{RG STUDY}

The description of the long-distance properties of the model (9) near the second order phase transition point is performed using the field-theoretical RG method [37]. Let us present the renormalization algorithm, developed for Lagrangian field theory. It is well known, that such a theory encounters with ultraviolet divergences, the re- 
moval of which is achieved within an appropriate renormalization procedure by a controlled rearangement of the perturbation theory series. The change of bare couplings $u_{0}, v_{0}$ and of the anisotropy constant $a_{0}$ under renormalization is described by the RG functions:

$$
\begin{aligned}
\beta_{u}(u, v) & =\left.\frac{\partial u}{\partial \ln \kappa}\right|_{0}, \beta_{v}(u, v)=\left.\frac{\partial v}{\partial \ln \kappa}\right|_{0}, \\
\zeta_{a}(u, v) & =\left.\frac{\partial \ln a}{\partial \ln \kappa}\right|_{0} .
\end{aligned}
$$

Here, $u, v, a$ are the renormalized couplings and anisotropy constant, respectively, $\kappa$ is the rescaling parameter, the notation $\left.\right|_{0}$ indicates differentiation at fixed bare parameters. The bare fields $\phi, \tilde{\phi}$, the mass $\mu_{0}$ and the Onsager kinetic coefficient $\lambda_{0}$ are related to the renormalized ones $\varphi, \tilde{\varphi} \mu$ and $\lambda$ by:

$$
\begin{array}{rlrl}
\phi & =Z_{\varphi}^{1 / 2} \varphi, & & \tilde{\phi}=Z_{\tilde{\varphi}}^{1 / 2} \tilde{\varphi}, \\
\mu_{0}^{2}=Z_{\mu^{2}} \mu^{2}, & & \lambda_{0}^{-1}=Z_{\lambda} \lambda^{-1},
\end{array}
$$

where $Z$-factors are dimensionless functions of renormalized parameters $m, u, v, a$. Their flows are defined by corresponding RG functions:

$$
\begin{aligned}
\gamma_{\phi}(u, v) & =\left.\frac{\partial \ln Z_{\varphi}}{\partial \ln \kappa}\right|_{0}, \\
\gamma_{\tilde{\phi}}(u, v) & =\left.\frac{\partial \ln Z_{\tilde{\varphi}}}{\partial \ln \kappa}\right|_{0}, \\
\bar{\gamma}_{\phi^{2}}(u, v) & =\left.\frac{\partial \ln Z_{\mu^{2}}^{-1}}{\partial \ln \kappa}\right|_{0}-\gamma_{\phi}, \\
\zeta(u, v) & =\left.\frac{\partial \ln Z_{\lambda}}{\partial \ln \kappa}\right|_{0} .
\end{aligned}
$$

Functions $\zeta$ and $\gamma_{\tilde{\phi}}$ are connected by the relation: $\zeta=$ $\left(\gamma_{\phi}-\gamma_{\tilde{\phi}}\right) / 2$.

The fixed points (FPs) $u^{*}, v^{*}$ of the RG transformation are defined as common zeroes of the $\beta$-functions:

$$
\beta_{u}\left(u^{*}, v^{*}\right)=0, \beta_{v}\left(u^{*}, v^{*}\right)=0 .
$$

A FP is stable, if the eigenvalues of the stability matrix, defined as: $B_{i j}=\partial \beta_{u_{i}}\left(u^{*}, v^{*}\right) / \partial \beta_{u_{j}}\left(u^{*}, v^{*}\right), u_{i}=\{u, v\}$ have positive real parts. In the case when the stable FP is physically accessible, i.e. can be reached starting from the initial values of the renormalized couplings (in our case, $u>0, v<0$ ), corresponds to the critical point of the system. The RG functions (12)-(15) taken at this point give the critical exponents of magnetic susceptibility, correlation length and relaxation time:

$$
\begin{aligned}
\gamma^{-1} & =1-\frac{\bar{\gamma}_{\phi^{2}}\left(u^{*}, v^{*}\right)}{2-\gamma_{\phi}\left(u^{*}, v^{*}\right)} \\
\nu_{\perp}^{-1} & =2-\bar{\gamma}_{\phi^{2}}\left(u^{*}, v^{*}\right)-\gamma_{\phi}\left(u^{*}, v^{*}\right) \\
z_{\perp} & =2+\zeta\left(u^{*}, v^{*}\right) .
\end{aligned}
$$

Note, that the following relations between the exponents describing the parallel and perpendicular correla- tion length and relaxation times hold [15]:

$$
\begin{aligned}
& \nu_{\|}=\nu_{\perp}\left(1-\frac{\zeta_{a}}{2}\right), \\
& z_{\|}=z_{\perp} /\left(1-\frac{\zeta_{a}}{2}\right) .
\end{aligned}
$$

To obtain the quantitative characteristics of the dynamical critical behavior of magnetic systems with extended impurities, we turn our attention to the RG functions derived in Ref. [18] in two-loop approximation:

$$
\begin{aligned}
\beta_{u} / u= & -\varepsilon+\frac{(m+8)}{6} u+2 v-\frac{(3 m+14)}{12} u^{2} \\
- & \frac{1}{12} u v\left[\frac{2}{3}(11 m+58)+(m-4) \frac{\varepsilon_{d}}{3\left(\varepsilon+\varepsilon_{d}\right)}\right] \\
- & v^{2} \frac{1}{144}\left[328+32 \frac{\varepsilon_{d}}{\varepsilon+\varepsilon_{d}}\right] \\
\beta_{v} / v= & -\varepsilon-\varepsilon_{d}+\frac{4}{3} v+\frac{m+2}{3} u-\frac{7}{6} v^{2} \\
- & v u \frac{m+2}{18}\left[11-\frac{\varepsilon_{d}}{\varepsilon+\varepsilon_{d}}\right]-\frac{5}{12} \frac{m+2}{3} u^{2} \\
\gamma_{\phi}= & \frac{1}{36} v^{2}+\frac{m+2}{36} v u+\frac{m+2}{72} u^{2} \\
\bar{\gamma}_{\phi^{2}}= & u \frac{m+2}{6}+\frac{1}{3} v-\frac{m+2}{6} u^{2}-24 v^{2} \\
- & \frac{m+2}{24} v u\left[6-\frac{\varepsilon_{d}}{\varepsilon+\varepsilon_{d}}\right] \\
\zeta_{a}= & \frac{1}{3} v-\frac{5}{36} v^{2}-\frac{(m+2)}{36} v u \\
\zeta= & -\frac{1}{3} v+\frac{(m+2)\left(6 \ln \frac{4}{3}-1\right)}{72} u^{2}+ \\
& \frac{(m+2)}{36} v u+\frac{5}{36} v^{2} .
\end{aligned}
$$

Here, $\varepsilon=4-d$ and the replica limit $n=0$ has been assumed. Putting in Eqs. (20)-(23), (25) $\varepsilon_{d}=0$ one regains the RG functions of the $m$-vector magnet with point-like defects [38, 39, 40].

The description of the critical behavior of magnets with extended impurities carried out in Refs. 15, 16, 18, 19] was based on the double expansion in two parameters $\varepsilon, \varepsilon_{d}$, considering both to be of the same order of magnitude. Being quite clear technically, such a statement causes however certain cautions. Indeed, taking the $\varepsilon$ as a small parameter is due to the fact that it deviates from the upper critical dimension, where the $\phi^{4}$ theory becomes asymptotically free [37], whereas $\varepsilon_{d}$ is the dimension of defect itself and obviously has a different physical origin. Therefore, it is desirable to search for an alternative way to analyze the RG functions (20)-(25). Fortunately, such an alternative exists and it is exploited in the field-theoretical approach to critical phenomena [41]. Namely, it consists in the analysis of minimal subtraction RG functions directly at dimension of space of interest, fixing $\varepsilon$ and solving the FP equations numerically [4]] (the so-called fixed dimension scheme). It was 
proposed [20, 21] to extend the approach of direct evaluation to the RG functions of the present model, i.e. to treat them directly at $d=3(\varepsilon=1)$ for different fixed values of the (non-integer) defect dimensionality $\varepsilon_{d}$. In our calculations (see Section IV], we will make use of both possibilities, exploiting $\varepsilon, \varepsilon_{d}$ expansion as well as working within the fixed dimension scheme.

In the field-theoretical RG approach the series expansions of the RG functions in powers of the coupling appear to be divergent; moreover, they are characterized by a factorial growth of the coefficients implying a zero radius of convergence [37]. To take into account the higher order contributions, the application of special tools of resummation is required [42]. In our previous paper 21], analyzing static critical behavior we applied the Chisholm-Borel resummation technique. Here, the Borel image of the initial function is extrapolated by a rational Chisholm [43] approximant $[K / L](x, y)$. One constructs this ratio of two polynomials of order $K$ and $L$ such that its truncated Taylor expansion is equal to that of the Borel image of the initial function. The resummed function is then calculated by an inverse Borel transform of this approximant. The details can be found in [44].

\section{THE RESULTS}

The critical behavior of the model (9) is influenced by the presence of the following FPs: the Gaussian FP $\mathbf{G}\left(u^{*}=v^{*}=0\right)$, the $O(m)$-symmetric $\mathrm{FP}$ of a pure magnet $\mathbf{P}\left(u^{*} \neq 0, v^{*}=0\right)$ and the random $\mathrm{FP} \mathbf{R}$ $\left(u^{*} \neq 0, v^{*} \neq 0\right)$ that governs disorder-induced critical behavior. A polymer FP with $u^{*}=0, v^{*}>0$ is not reachable from the initial values of couplings and therefore is out of interest for our study. Depending on the values of global parameters $d, \varepsilon_{d}, m$ one of the above FPs is stable and in the asymptotic limit governs the criticality. However, as we will show in the section $\nabla$ an approach to the asymptotic limit and hence the effective critical behavior is influenced by all the FPs.

For the $d=3$ magnets considered here, it is the crossover between FPs $\mathbf{P}$ and $\mathbf{R}$ that corresponds to the change of the universal properties of an $m$-vector magnet upon dilution by $\varepsilon_{d}$-dimensional defects. According to the generalized Harris criterion, this crossover occurs at certain marginal value $m_{c}\left(\varepsilon_{d}\right)$. At $m>m_{c}$, the FP $\mathbf{P}$ is stable, indicating no change in critical behavior, whereas for $m<m_{c}$ one finds the stability of the FP $\mathbf{R}$, displaying the fact of relevance of disorder. From the generalized Harris criterion one can obtain the marginal value $\varepsilon_{d}^{\text {marg }}$ as function of $m$. Using the six-loop results 28 for the correlation length critical exponent $\nu_{p}(m)$ of the pure $m$-vector magnet, one obtains: $\varepsilon_{d}^{\operatorname{marg}}(m=1)=-0.173 ; \varepsilon_{d}^{\operatorname{marg}}(m=2)=0.016$; $\varepsilon_{d}^{\operatorname{marg}}(m=3)=0.172 ; \varepsilon_{d}^{\operatorname{marg}}(m=4)=0.300$. These estimates are shown in Fig. [1 by filled squares connected by a solid line. The figure may serve as a phase diagram of an $m$-vector magnet with extended impurities: the new critical behavior is expected in the region of $\varepsilon_{d}-m$ plane denoted as "Diluted". In the region denoted "Pure" the asymptotic critical behavior does not indicate presence of defects, however the effective critical behavior does. Below, we will perform an analysis of the critical dynamics for both regions of the phase diagram.

\section{A. Fixed $d=3$ scheme}

We start from the RG $\beta$-functions (20), (21): fixing the value $\varepsilon=1$ (i.e. $d=3$ ) and treating $\varepsilon_{d}$ as a varying parameter, we look for the common zeros of the resummed functions $\beta_{u}$ and $\beta_{v}$. The numerical values of the stable FP coordinates obtained for the three-dimensional $m$-component magnets with $m=1,2,3,4$ can be found in our previous paper [21].

To calculate the values of the critical exponents $z_{\perp}$ we substitute Eq. (25) for $\zeta$ in Eq. (18), apply the resummation procedure for the resulting series, and, finally, estimate them at the stable FP. Note, that because the function $\zeta$ is not symmetric in variables $u$ and $v$ (namely, it does not contain term linear in $u$ ) the Chisholm approximant chosen for its analytic continuation differs from those chosen for the $\beta$-functions. The value of critical exponent $z_{||}$is obtained using the relation (19).

TABLE I: The values of dynamical critical exponents of three-dimensional $m$-component magnets at different fixed values of extended defect dimension $\varepsilon_{d}$.

\begin{tabular}{|c|c|c|c|c|c|c|c|c|}
\hline & & $m=1$ & & $m=2$ & & $m=3$ & & $m=4$ \\
\hline$\varepsilon_{d}$ & $z_{\perp}$ & $z_{\|}$ & $z_{\perp}$ & $z_{\|}$ & $z_{\perp}$ & $z_{||}$ & $z_{\perp}$ & $z_{\|}$ \\
\hline 0 & 2.172 & - & 2.065 & - & 2.062 & - & 2.058 & - \\
\hline 0.1 & 2.248 & 2.094 & 2.079 & 2.068 & 2.062 & 2.062 & 2.058 & 2.058 \\
\hline 0.2 & 2.302 & 2.111 & 2.135 & 2.073 & 2.062 & 2.062 & 2.058 & 2.058 \\
\hline 0.3 & 2.340 & 2.127 & 2.183 & 2.074 & 2.095 & 2.063 & 2.058 & 2.058 \\
\hline 0.4 & 2.367 & 2.142 & 2.223 & 2.081 & 2.133 & 2.063 & 2.083 & 2.061 \\
\hline 0.5 & 2.386 & 2.156 & 2.256 & 2.087 & 2.166 & 2.064 & 2.111 & 2.062 \\
\hline 0.6 , & 2.399 & 2.169 & 2.284 & 2.092 & 2.195 & 2.067 & 2.138 & 2.065 \\
\hline 0.7 & 2.408 & 2.181 & & 2.096 & 2.219 & 2.069 & 2.162 & 2.067 \\
\hline 0.8 & 2.413 & 2.194 & 2.326 & 2.100 & 2.241 & 2.070 & & 2.069 \\
\hline 0.9 & 2.416 & 2.206 & 2.342 & 2.104 & 2.260 & 2.072 & 2.203 & 2.070 \\
\hline 1.0 & 2.418 & 2.217 & 2.356 & 2.107 & 2.276 & 2.073 & 2.219 & 2.071 \\
\hline 1.1 & 2.418, & 2.228 & 2.368 & 2.109 & 2.290 & 2.073 & 2.233 & 2.071 \\
\hline
\end{tabular}

In Table \we give the obtained results for critical exponents $z_{\|}, z_{\perp}$ of three-dimensional $m$-component magnets. The case $\varepsilon_{d}=0$ corresponds to point-like defects. As it was already noted in the Introduction, for this case only the Ising model $(m=1)$ is influenced by disorder, and thus for systems with $m>1$ the values of the critical exponents are not altered by disorder. Here, one can compare our result with the dynamic exponent of the pure and diluted Ising models, as shown in Tables 【III When $\varepsilon_{d}$ increases, for $m=2,3,4$ the critical exponents 
remain constant and equal to the corresponding exponents of the pure model, until $m$ becomes $m_{c}$ for given $\varepsilon_{d}$ and for $m>m_{c}$ the values of exponents start to increase because they take their new values belonging to the disordered universality class.

TABLE II: The value of dynamical critical exponent $z$ for pure three-dimensional Ising model. MC: Monte Carlo simulations; exp: experimental estimates for $\mathrm{FeF}_{2}$. Theoretical estimates, $\sim \varepsilon^{2}\left(\varepsilon^{3}\right)$ : direct substitution $\varepsilon=1$ into $\sim \varepsilon^{2}\left(\varepsilon^{3}\right)$ expansions; $\sim \varepsilon^{3}$, res: resummation of $\varepsilon^{3}$ expansion; 4 loops, res: resummation of four-loop massive $3 \mathrm{~d} R \mathrm{R}$ functions.

\begin{tabular}{lcc}
\hline \hline & Method & Result \\
\hline Ref. [46] & MC & $2.04 \pm 0.03$ \\
Ref. [47] & MC & $2.06 \pm 0.02$ \\
Ref. [48] & MC & $2.03 \pm 0.01$ \\
Ref. [49] & MC & $2.032 \pm 0.004$ \\
Ref. [50] & MC & $2.055 \pm 0.003$ \\
Ref. [51] & exp & $2.1 \pm 0.1$ \\
Ref. [18] & $\sim \varepsilon^{2}$ & 2.014 \\
Eq. [32] & $\sim \varepsilon^{3}$ & 2.024 \\
Eq. [32] & $\sim \varepsilon^{3}$, res & 2.012 \\
Ref. [52] & 4 loops, res & 2.017 \\
\hline \hline
\end{tabular}

TABLE III: The value of dynamical critical exponent $z$ for three-dimensional Ising model with point-like uncorrelated defects. MC: Monte Carlo simulations; exp: experimental estimates for $\mathrm{Fe}_{0.9} \mathrm{Zn}_{0.1} \mathrm{~F}_{2}$. Theoretical estimates, $\sim \varepsilon^{1 / 2}$ : direct substitution $\varepsilon=1$ into $\varepsilon^{1 / 2}$-expansions; 2 loops, res: resummation of two-loop massive $3 \mathrm{~d}$ RG functions; $\sim \varepsilon^{1 / 2}\left(\varepsilon^{3 / 2}\right)$ res: substitution of the two (three)-loop resummed FP coordinates into the two-loop expansions for $z$.

\begin{tabular}{lcc}
\hline \hline & Method & Result \\
\hline Ref. [53] & MC & $2.13 \pm 0.05$ \\
Ref. [54] & MC & $2.4 \pm 0.1$ \\
Ref. [55] & MC & $2.62 \pm 0.07$ \\
Ref. [56] & exp & $2.18 \pm 0.10$ \\
Ref. [57] & $\sim \varepsilon^{1 / 2}$ & 2.336 \\
Ref. [53] & 2 loops, res & 2.237 \\
Ref. [38] & $\sim \varepsilon^{1 / 2}$, res & 2.023 \\
Ref. [58] & $\sim \varepsilon^{3 / 2}$, res & 2.180 \\
\hline \hline
\end{tabular}

An interesting feature of the data shown in Table $\prod$ is that for each fixed $\varepsilon_{d}$ the relation $z_{\|}<z_{\perp}$ holds. One can give the following physical interpretation to this fact. As it has been noted for isotropic systems [32], the dynamical critical exponent $z$ is proportional to the pair correlation function critical exponents $\eta$ :

$$
z=2+c \eta \text {. }
$$

For the systems we consider here the critical exponents $\eta_{\perp}$ and $\eta_{\|}$, that characterize the behavior of the pair spinspin correlation function in the directions, perpendicular and parallel to the extended defects, are distinguished [15]. As far as the extended defects cut interacting bonds of spins perpendicular to the extended-defect direction, in the parallel direction the fluctuations are stronger and therefore $\eta_{\|}<\eta_{\perp}$ and thus, by Eq. (26) with $z_{\|}, z_{\perp}$ we obtain the confirmation to our results. However, in the anisotropic case the coefficient $c$ in Eq. (26) is also direction dependent. Thus the above argumentation is rather of qualitative nature to give a hint to physical interpretation of the observed relation $z_{\|}<z_{\perp}$.

In our previous papers 20, 21] we have also touched the question of existence of an upper marginal value for the defect dimensionality $\varepsilon_{d}$. In our analysis, we observe the disappearance of a stable reachable FP for $\varepsilon_{d}$ slightly above 1 . The following physical interpretation was proposed: extended defects of large dimension (e.g. parallel planar defects with $\varepsilon_{d}=2$ ), may divide the system into non-interacting regions and thus inhibit ferromagnetic order. Recently in Refs. 45] the Ising magnet with spin interaction bonds, correlated in 2 dimensions and randomly distributed in the perpendicular direction, was studied. Although such a system differs from those considered here, both models possesses a number of common features. In particular, the smearing of a phase transition due to the presence of planar defects was predicted and explained by the existence - although rare - of infinite spatial regions, which are free of defects and therefore may be locally in the ordered phase.

To confirm the obtained results, we have also tried to apply the Padé-Borel resummation to estimate the values for $z_{\perp}, z_{\|}$. In this procedure, one rewrites the twovariable series (20), (21) in terms of a resolvent series 59] of one variable and then applies a Padé approximant for its analytic continuation. We do not present the results obtained, they give rather close numbers to those in Table III and reproduce the same behavior.

\section{B. $\varepsilon, \varepsilon_{d}$-expansion}

Another possibility to obtain estimates for dynamic critical exponents of the $m$-vector magnet with extended defects is to resum the double $\varepsilon, \varepsilon_{d}$ expansions obtained in the two-loop approximation in Ref. [18]. For $m<m_{c}$, the random FP $\mathbf{R}$ is stable and the expression for $z_{\perp}$ and anisotropy function $\zeta_{\alpha}$ for $m \neq 1$ read [18]: 


$$
\begin{aligned}
z_{\perp} & =2-\frac{m+2}{4(m-1)} \varepsilon+\frac{(m+8)}{8(m-1)} \tilde{\varepsilon}+\left\{-4(m+2)\left[5 m^{2}+42 m+112-192(m-1) \ln (4 / 3)\right] \varepsilon^{2}\right. \\
& -4(m+2)\left[27 m^{2}-264 m-240+576(m-1) \ln (4 / 3)\right] \varepsilon \tilde{\varepsilon} \\
& \left.+\left[59 m^{3}-528 m^{2}-2928 m-896+1728(m+2)(m-1) \ln (4 / 3)\right] \tilde{\varepsilon}^{2}\right\}\left(1024(m-1)^{3}\right)^{-1} \\
\zeta_{\alpha} & =\frac{(m+2)}{4(m-1)} \varepsilon+\frac{m+8}{8(m-1)} \tilde{\varepsilon} \\
& +\left[-4(m+2)\left(5 m^{2}+10 m+144\right) \varepsilon^{2}-4(m+2)\left(27 m^{2}-168 m-336\right) \varepsilon \tilde{\varepsilon}\right. \\
& \left.+\left(59 m^{3}-240 m^{2}-2640 m-1472\right) \tilde{\varepsilon}^{2}\right]\left[1024(m-1)^{3}\right]^{-1}, \quad \tilde{\varepsilon}=\varepsilon+\varepsilon_{d} .
\end{aligned}
$$

Whereas for $m>m_{c}$, the pure FP $\mathbf{P}$ is stable. Therefore the critical behavior is isotropic and the dynamic exponent $z$ reads:

$$
z=2+0.363 \frac{(m+2) \varepsilon^{2}}{(m+8)^{2}} .
$$

As it is known 60], due to degeneracy of the $\beta$ functions of the weakly diluted Ising model with pointlike defects, the usual $\varepsilon$-expansion for critical exponents turns into the $\sqrt{\varepsilon}$-expansion. The same holds for the extended defects: indeed, the case $m=1$ is to be analyzed separately as far as expressions (27), (28) contain poles at $m=1$, and one arrives [18] at the $\sqrt{\varepsilon}$-expansion for the critical exponents. In particular, the corresponding expressions for $z$ can be given only to the lowest nontrivial order. Moreover, the $\sqrt{\varepsilon}$-expansion does not allow for a reliable numerical estimate [40]. Therefore, for the disordered Ising model, the fixed dimension scheme considered in the subsection IVA remains the only way to get numerical estimates.

To get the numerical estimate for $m_{c}$ in the frames of $\varepsilon, \varepsilon_{d}$-expansion we substitute into the modified Harris criterion the five-loop $\varepsilon$ - expansion for the correlation length critical exponent $\nu_{p}$ for pure $m$-component model, given in [61] and obtain the following expansion:

$$
\begin{aligned}
m_{c} & =\left(-4+8 \frac{\tilde{\varepsilon}}{\varepsilon}-2.50000 \frac{\tilde{\varepsilon}^{2}}{\varepsilon}-1.500000 \tilde{\varepsilon}-2.448919 \frac{\tilde{\varepsilon}^{3}}{\varepsilon}+3.014557 \tilde{\varepsilon}^{2}+4.141561 \varepsilon \tilde{\varepsilon}-1.682130 \frac{\tilde{\varepsilon}^{4}}{\varepsilon}-\right. \\
& -14.12940 \varepsilon^{2} \tilde{\varepsilon}-0.5736055 \varepsilon \tilde{\varepsilon}^{2}+7.657623 \tilde{\varepsilon}^{3}+55.57104 \varepsilon^{3} \tilde{\varepsilon}-16.25104 \varepsilon^{2} \tilde{\varepsilon}^{2}-37.62878 \varepsilon \tilde{\varepsilon}^{3}+ \\
& \left.+22.53257 \tilde{\varepsilon}^{4}-3.345417 \frac{\tilde{\varepsilon}^{5}}{\varepsilon}\right)\left(2-\frac{\tilde{\varepsilon}}{\varepsilon}\right)^{-1}, \quad \tilde{\varepsilon}=\varepsilon+\varepsilon_{d}
\end{aligned}
$$

Putting here $\tilde{\varepsilon}=\varepsilon$ (i.e $\varepsilon_{d}=0$ ) one recovers the $\varepsilon$ expansion for $m_{c}$ of the model with point-like uncorrelated defects [13].

To estimate $m_{c}$ numerically for different fixed values of $d, \varepsilon_{d}$, one should apply a resummation. As it is known [13, 62] already simple Padé-analysis gives convergent results for marginal dimensions. In the Padé analysis [63], it is known that the best convergence is achieved along the main diagonal of a Padé table, therefore we make use of the diagonal [2/2] Padé-approximant. In the threedimensional case $(\varepsilon=1)$ it gives: $m_{c}\left(\varepsilon_{d}=0\right)=1.92$ (which is in a good agreement with the known six-loop results for the point-like disorder $m_{c}=1.942 \pm 0.026$ [12] and $m_{c}=1.912 \pm 0.004$ [13] $), m_{c}\left(\varepsilon_{d}=0.1\right)=2.48$, $m_{c}\left(\varepsilon_{d}=0.2\right)=3.10, m_{c}\left(\varepsilon_{d}=0.3\right)=3.77, m_{c}\left(\varepsilon_{d}=\right.$ $0.4)=4.54$. The results obtained are plotted by the dotted line in Fig. [1]
To estimate dynamical critical exponents in the FP $\mathbf{R}$ we applied the Chisholm-Borel resummation technique to the functions (27), (28) treating them as series in two variables $\varepsilon, \varepsilon_{d}$ and using the Chisholm approximant. In the corresponding two-loop approximation the dynamical critical exponents in the pure FP $\mathbf{P}$ is given by Eq. (29) and the expression is too short to be resummed. Therefore it has been estimated at $\varepsilon=1$ and different $m$ by a direct substitution. The results are presented in Table IV They qualitatively confirm our conclusions made on the basis of fixed dimension technique in subsection IVA (see Table III). The discrepancy between the data of the two techniques may serve also as an estimate for accuracy of the perturbation schemes applied.

Let us note, that currently the three-loop series for the exponent $z$ of the pure $m$-vector magnet model A 
dynamics is available. The expression found in [64 reads:

$$
z=2+0.726(1-\varepsilon \cdot 0.1885) \eta .
$$

Substituting into (31) the $\varepsilon$-expansion for $\eta[61]$, one gets:

$$
z=2+0.363 \frac{(m+2) \varepsilon^{2}}{(m+8)^{2}}+\left(0.09075 \frac{(m+2)\left(-m^{2}+56 m+272\right)}{(m+8)^{4}}-0.363 \frac{0.1885 m+0.3770}{(m+8)^{2}}\right) \varepsilon^{3} .
$$

Numerical estimates of this series based on the PadéBorel resummation are given in the Table \ for $m=1$ and compared with values found by other approaches.

TABLE IV: The dynamical critical exponents of $m$-component model with extended impurities, obtained on the base of resumming the double $\varepsilon, \varepsilon_{d}$-expansion (Eq. (27)).

\begin{tabular}{cccccccc}
\hline \hline & \multicolumn{3}{c}{$m=2$} & \multicolumn{3}{c}{$m=3$} & \\
\hline$\varepsilon_{d}$ & $z_{\perp}$ & $z_{\|}$ & $z_{\perp}$ & $z_{\|}$ & $z_{\perp}$ & $z_{\|}$ \\
0 & 2.0145 & - & 2.0150 & - & 2.0151 & - \\
0.1 & 2.021 & 2.0147 & 2.0150 & 2.0150 & 2.0151 & 2.0151 \\
0.2 & 2.046 & 2.0150 & 2.0150 & 2.0150 & 2.0151 & 2.0151 \\
0.3 & 2.067 & 2.0151 & 2.0511 & 2.020 & 2.0151 & 2.0151 \\
0.4 & 2.175 & 2.094 & 2.088 & 2.050 & 2.024 & 1.984 \\
0.5 & 2.209 & 2.110 & 2.129 & 2.081 & 2.064 & 2.020 \\
0.6 & 2.238 & 2.122 & 2.167 & 2.101 & 2.114 & 2.054 \\
0.7 & 2.270 & 2.138 & 2.210 & 2.120 & 2.158 & 2.085 \\
0.8 & 2.287 & 2.146 & 2.247 & 2.140 & 2.195 & 2.113 \\
0.9 & 2.315 & 2.157 & 2.282 & 2.153 & 2.244 & 2.126 \\
1.0 & 2.333 & 2.171 & 2.317 & 2.169 & 2.279 & 2.141 \\
1.1 & 2.354 & 2.175 & 2.346 & 2.176 & 2.314 & 2.164 \\
\hline \hline
\end{tabular}

\section{EFFECTIVE CRITICAL BEHAVIOR}

The previous discussion concerns the asymptotic critical dynamics of the model (9). Here we discuss the effective critical behavior which is observed in approaching the critical point $T_{c}$ 29]. This behavior is characterized by effective critical exponents governing scaling laws when $T_{c}$ still is not reached. The calculation of effective critical exponents for models with extended impurities was not considered so far in dynamics as well as in the statics. However these exponents are mainly observed in numerical and real experiments, which may be outside the asymptotic region.

The effective critical exponents are defined as logarithmic derivatives of the appropriate quantities of interest with respect to the reduced temperature $t=\left|T-T_{c}\right| / T_{c}$ [29]. For instance, effective critical exponent for perpen- dicular correlation length is defined as:

$$
\nu_{\perp}^{\mathrm{eff}}(t)=-\frac{\mathrm{d} \ln \xi_{\perp}(t)}{\mathrm{d} \ln t} .
$$

In the limit $T \rightarrow T_{c}$ the effective exponent coincides with the asymptotic one $\nu_{\perp}^{\text {eff }}=\nu_{\perp}$. Within the fieldtheoretical RG approach effective exponents are calculated using the values of couplings (solution of flow equation) depending on the flow parameter $\ell$. For instance, effective exponents for correlation length and relaxation time perpendicular to the extended impurities direction are defined as:

$$
\begin{aligned}
& 1 / \nu_{\perp}^{\mathrm{eff}}(\ell)=2-\bar{\gamma}_{\phi^{2}}(u(\ell), v(\ell))- \\
& -\gamma_{\phi}(u(\ell), v(\ell))+\ldots ; \\
& z_{\perp}^{\mathrm{eff}}(\ell)=2+\zeta(u(\ell), v(\ell))+\ldots
\end{aligned}
$$

The flow parameter $\ell$ is related to the temperature distance to the critical point via the inverse correlation length. Therefore the dependence of the effective exponents on the flow parameter corresponds to a dependence on the reduced temperature [65]. In (34) and (35) the parts denoted by dots come from the change of the amplitude part of the perpendicular correlation length and critical relaxation time correspondingly. In the subsequent calculations we will neglect this part, since the contribution of the amplitude function to the crossover seems to be small [66].

Solving the flow equation for different initial conditions we can obtain different flows in the space of couplings. We choose these condition near the Gaussian FP $(\mathbf{G})$, expecting that couplings are small in the background region. In flow equations (10) we use the $\beta$-function (20), (21) at fixed $d$ resummed by the Chisholm-Borel method. This allows us to investigate also the case of Ising systems $(m=1)$, for which the $\beta$-function are degenerated on the one-loop level and the $\sqrt{\varepsilon}$-expansion does not allow for numerical estimates [40].

The RG flows at $m=1$ for the two most interesting cases $\varepsilon_{d}=0$ and $\varepsilon_{d}=1$ are shown in the Fig. [2] (dashed lines for $\varepsilon_{d}=0$ and solid lines for $\varepsilon_{d}=1$ ). The first case corresponds to the point defects, whereas the second one corresponds to the lines of defects. We consider the same initial condition for both cases with a small (flows 1 and $1^{\prime}$ ) and with a large (flows 2 and $2^{\prime}$ ) value of the ratio $v / u$. The ratio $v / u$ defines the degree of disorder, thus 


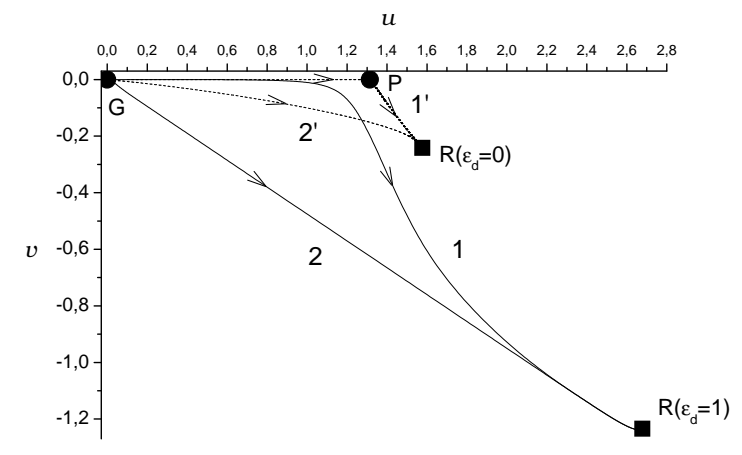

FIG. 2: Flows for the case $m=1$. Dashed lines correspond to the case of uncorrelated point defects, $\varepsilon_{d}=0$, while solid lines correspond to system with extended lines of defects, $\varepsilon_{d}=1$. The initial values are the same for curves 1 and $1^{\prime}$ (small ratio $\Delta / u$ ) and for 2 and $2^{\prime}$ (large ratio $v / u$ ).

we can observe difference in the behavior of systems with low and high dilution.

Using the flows given in the Fig. 2 we can obtain static and dynamic effective exponents. Below, we choose to display the exponents $\nu_{\perp}^{\mathrm{eff}}$ and $z_{\perp}^{\mathrm{eff}}$. Due to Eq. (19) the parallel effective exponents $\nu_{\|}^{\text {eff }}$ and $z_{\|}^{\text {eff }}$ have qualitatively similar behavior. The deviations of effective exponents from the mean-field values $z_{\perp}^{\text {eff }}-2$ and $\nu_{\perp}^{\text {eff }}-1 / 2$ corresponding to flows of Fig. 2 are given in the Fig. 3 and Fig. 4 respectively. First we compare the behavior of the effective exponents of the uniaxial $m=1 \mathrm{mag}$ nets at $\varepsilon_{d}=0$ and $\varepsilon_{d}=1$. As it can be seen from the Figs. 3 and 4 even a weak dilution by lines of defects leads to a faster crossover to the new universality class (curves 1 in Figs. 3 and 4 ) and the effective exponents are not influenced by the pure FP P. Such a behavior qualitatively differs from that at dilution by point-like impurities, where effective exponents corresponding to the pure FP $\mathbf{P}$ are observed in a relatively wide region (curves 1' in both figures). Note that the dynamical critical exponent $z_{\perp}^{\text {eff }}$ at $\varepsilon_{d}=1$ approaches its asymptotics from above. Such a nonmonotonic behavior is typical in statics for system with point-like defects [1, 40]. However we do not observe nonmonotonic behavior for $\nu_{\perp}^{\text {eff }}$. It may be a feature of static effective critical exponents for system with extended impurities or consequence of our approximation.

We also consider static and dynamic effective behavior for different values of order parameter dimension. Fig. 5 and Fig. [6] present the $z_{\perp}^{\text {eff }}-2$ and $\nu_{\perp}^{\text {eff }}-1 / 2$ correspondingly obtained for the same initial conditions and different values $m=1,2,3,4$. The nonmonotonic character of dependence of exponents on the logarithm of flow parameter is observed only for $z_{\perp}^{\text {eff }}$ at $m=1$.

\section{CONCLUSIONS}

The present study is dedicated to providing the reliable numerical estimates for the dynamical critical exponents

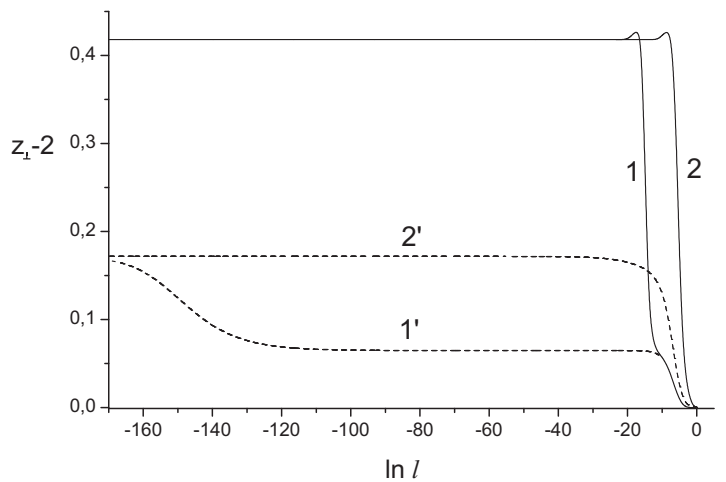

FIG. 3: Behavior of the dynamical effective exponent $z_{\perp}^{\text {eff }}$ for $m=1$ in the case of $\varepsilon_{d}=0$ (dashed lines) and $\varepsilon_{d}=1$ (solid lines). Curves correspond to flows of Fig 2

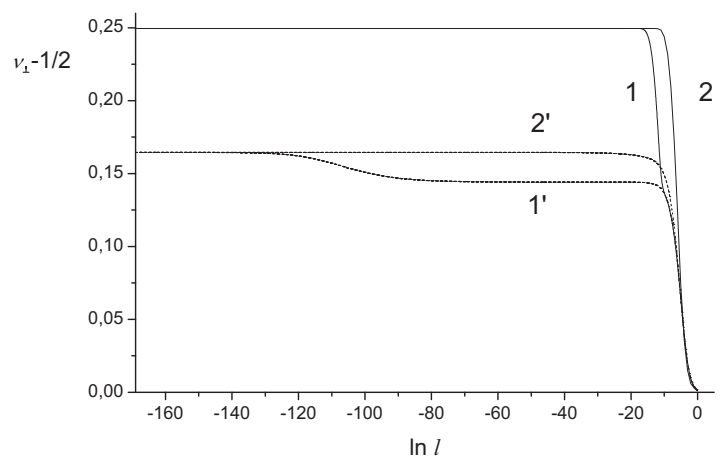

FIG. 4: Behavior of effective critical exponent of perpendicular correlation length $\nu_{\perp}^{\text {eff }}$ for $m=1$ in the case of $\varepsilon_{d}=0$ (dashed lines) and $\varepsilon_{d}=1$ (solid lines). Curves correspond to flows of Fig[2]

of magnetic systems with $\varepsilon_{d}$-dimensional non-magnetic impurities of parallel orientation. The presence of such impurities leads to anisotropy in the systems; thus, two correlation lengths, parallel and perpendicular to the extended defects exist, which diverge in the vicinity of critical temperature with different exponents. The dynamic

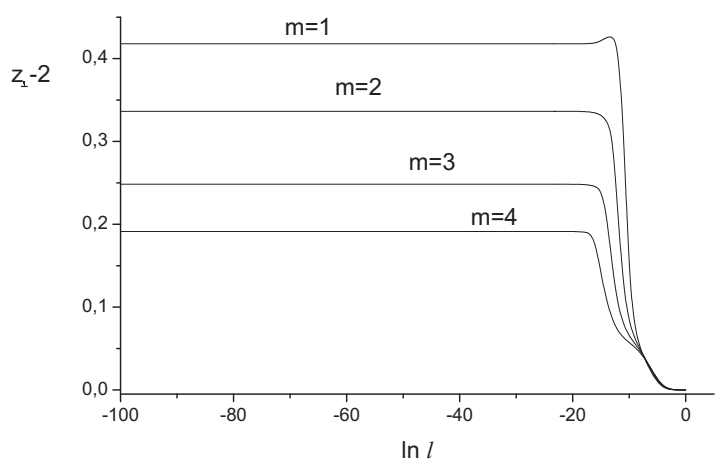

FIG. 5: Behavior of dynamical effective exponent $z_{\perp}^{\text {eff }}$ for system with extended impurities with $\varepsilon_{d}=1$ and different $m$. Initial conditions are the same in all cases (small ratio $v / u$ ). 


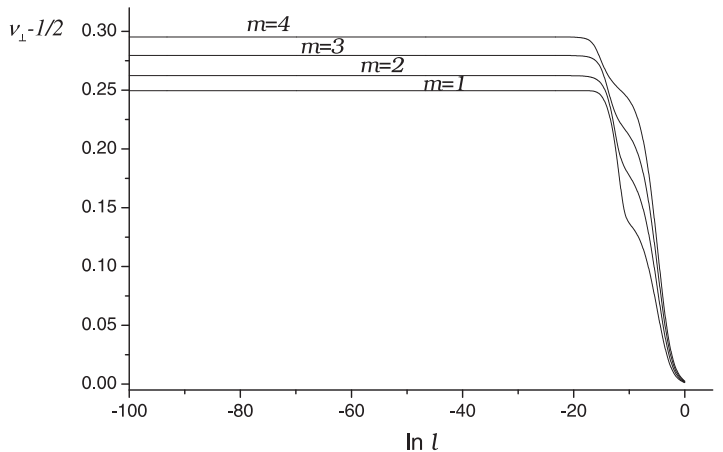

FIG. 6: Behavior of effective critical exponent of perpendicular correlation lenght $\nu_{\perp}^{\text {eff }}$ for system with extended impurities with $\varepsilon_{d}=1$ and different $m$. Initial conditions are the same in all cases (small ratio $v / u$ ).

critical behavior is modified as well, so that two times of critical relaxation in directions parallel and transverse to extended impurities appear. The former results obtained for such systems are based on the double expansion in parameters $\varepsilon, \varepsilon_{d}$ and thus are rather of a qualitative character.

An example of related systems is given by magnets with extended randomly oriented defects: these can be described by the model of Weinrib and Halperin [23]. There, the distribution of defects is characterized by the pair correlation function, falling off with distance for large separations according to a power law. The influence of such correlated defects on criticality was a subject of several theoretical studies [23, 24] and predicted new universal critical behavior found recently its confirmation in the MC simulations [25].

We applied the resummation to the $R G$ functions of the model, obtained in the minimal subtraction scheme 16, 18], treating them directly for fixed $d=3$ and fixed parameter $\varepsilon_{d}$. The case $\varepsilon_{d}=0$ describes pointlike quenched disorder and reproduces well-known results. For $\varepsilon_{d}>0$ it was found, that the relation $z_{\perp}>z_{\|}$ holds for every $\varepsilon_{d}$ and $m$, which is connected to the fact, that the extended defects cut interacting paths of spins perpendicular to the extended-defect direction, so in the parallel direction the fluctuations are stronger.

The data of Tables I IV give numerical values of the dynamical critical exponents for the model A dynamics of the $m$-vector magnets with extended $\varepsilon_{d}$-dimensional defects. Together with possible scenarios of the effective critical behavior discussed in the Section $\nabla$ it should facilitate experimental studies of the influence of extended defects on criticality. As we already noted, the point defects change the universality class at $d=3$ only for the Ising magnets $(m=1)$. Values of the critical exponent $z$ of the pure and the diluted (by point-like impurities) Ising model are given in Tables III III respectively. Comparing the data in these tables one sees an increase of the exponent for the diluted model with respect to the pure one. This corresponds to a stronger divergency of the relaxation time (increase of the critical slowing-down effects). Further, comparing Tables III and $\prod$ one sees that extended impurities make this effect more pronounced, leading to further increase of both exponents $z_{\perp}, z_{\|}$. However this increase (of the order of 8 $\%$ if one compares $z$-exponents for point and line defects) is not dramatic to make MC simulations impossible due to enhanced critical slowing down. On the other hand, the same order of difference in critical exponents values holds also for the static exponents [20, 21]. Therefore, we expect that the predicted change in the critical behavior of three dimensional magnets with extended impurities is within current experimental accuracy. Although it is more difficult to extract dynamical critical exponents from simulations to the same accuracy, it would be worthwhile to look for them (i) in the cases of different defect dimensionality and (ii) in the non-asymptotic regime.

\section{Acknowledgements}

We thank the Austrian Fonds zur Förderung der wissenschaftlichen Forschung, project No.16574-PHY which supported in part this research. V.B. thanks the W. Macke Stiftung for enabling her research stay in Linz.
[1] R. Folk, Yu. Holovatch, T. Yavors'kii, Physics - Uspekhi 46169 (2003) [Uspekhi Fizicheskikh Nauk 173 175], preprint cond-mat/0106468

[2] A. Pelissetto and E. Vicari, Phys. Rep. 368, 549 (2002).

[3] Yu. Holovatch, V. Blavats'ka, M. Dudka, C. von Ferber, R. Folk, and T. Yavors'kii. Int. J. Mod. Phys. B 16, 4027 (2002).

[4] D.P. Belanger, Brazilian Journ. Phys. 30, 682 (2000); preprint cond-mat/0009029

[5] H. Yamamoto, H. Onodera, K. Hosoyama, T. Masumoto, and H. Yamauchi, J. Magn. Magn. Mater. 31-34, 1579 (1983); K. Winschuh and M. Rosenberg, J. Appl. Phys. 61, 4401 (1987); G. K. Nicolaides, G. C. Hadjipanayis, and K. V. Rao, Phys. Rev. B 48, 12759 (1993);
P. D. Babu and S. N. Kaul, J. Phys.: Cond. Matt. 9, 7189 (1997).

[6] A. Perumal, V. Srinivas, K. S. Kim, S. C. Yu, V. V. Rao, and R. A. Dunlap, J. Magn. Magn. Mater. 233, 280 (2001).

[7] M. Fähnle, G Herzer, H. Kronmüller, R. Meyer, M. Saile, and T. Egami, J. Magn. Magn. Mater. 38, 240 (1983); S. N. Kaul, Phys. Rev. B 38, 9178 (1988); S. N. Kaul and Ch. V. Mohan, Phys. Rev. B 50, 6157 (1994).

[8] W.-U. Kellner, T. Albrecht, M. Fähnle, and H. Kronmüller, J. Magn. Magn. Mater. 62, 169 (1986).

[9] O. Boxberg and K. Westerholt, Phys. Rev. B 50, 9331 (1994).

[10] K. Westerholt and G. Sobotta, J. Phys. F 13, 2371 
(1983); K. Westerholt, H. Bach, and R. Römer, J. Magn. Magn. Mater. 45, 252 (1984); K. Westerholt, Physica 130B, 533 (1985); K. Westerholt, J. Magn. Magn. Mater. 66, 253 (1987).

[11] A.B. Harris, J. Phys. C 7, 1671 (1974).

[12] C. Bervillier, Phys. Rev. B, 34, 8141 (1986).

[13] Yu. Holovatch, M. Dudka, and T. Yavors'kii, J. Phys. Studies 5, 233 (2001).

[14] R. Balien, M. Kleman, and J. Poirier, Physics of Defects, Les Houches, 1980 (North Holland, Amsterdam,1981).

[15] S.M. Dorogovtsev, Fiz. Tverd. Tela (Leningrad) 22, 321 (1980) [Sov. Phys.-Solid State 22, 188 (1980)]; Fiz. Tverd. Tela (Leningrad) 22, 3659 (1980) [Sov. Phys.Solid State 22, 2141 (1980)].

[16] D. Boyanovsky and J.L. Cardy, Phys. Rev. B 26, 154 (1982); Phys. Rev. B 27, 6971 (1983)

[17] V.V. Prudnokov, J. Phys. C 163685 (1983).

[18] I. D. Lawrie and V.V. Prudnikov, J. Phys. C: Solid State Phys. 17, 1655 (1984).

[19] Y. Yamazaki, A. Holz, M. Ochiai, and Y. Fukuda, Physica A 150, 576 (1988); Y. Yamazaki, A. Holz, M. Ochiai, and Y. Fukuda, Phys. Rev. B 33, 3460 (1986); Physica A 136, 303 (1986); Y. Yamazaki, M. Ochiai, A. Holz, and Y. Fukuda, Phys.Rev. B 33, 3474 (1986).

[20] V. Blavats'ka, C. von Ferber, Yu. Holovatch, Acta Phys. Slovaca 52317 (2002).

[21] V. Blavats'ka, C. von Ferber, Yu. Holovatch, Phys. Rev. B. 67094404 (2003).

[22] A. A. Fedorenko, Phys. Rev. B 69, 134301 (2004).

[23] A. Weinrib and B.I. Halperin, Phys. Rev. B 27, 413 (1983).

[24] V.V. Prudnikov, P.V. Prudnikov, and A.A. Fedorenko, J. Phys. A 32, L399 (1999); J. Phys. A 32, 8587 (1999); Phys. Rev. B 62, 8777 (2000); E. Korutcheva and F. Javier de la Rubia, Phys. Rev. B 58, 5153 (1998); V. Blavats'ka, C. von Ferber, Yu. Holovatch, Phys. Rev. E 64, 041102 (2001).

[25] H.G. Ballesteros, G. Parisi Phys. Rev. B 60, 12912 (1999); V.V. Prudnikov, P.V. Prudnikov, S.V. Dorofeev, V.Yu. Kolesnikov, Condens. Matter. Phys. 8 (2005), to appear.

[26] J.C. Lee and R.L. Gibbs, Phys. Rev. B 45, 2217 (1992); L. De Cesare, Phys. Rev. B 49, 11742 (1994); A.L. Korzhenevskii, K. Herrmanns, and W. Schirmacher, Phys. Rev. B 53, 14834 (1996).

[27] Y. Gefen, B.B. Mandelbrot, A. Aharony, Phys. Rev. Lett. 45, 855 (1980); Y.K. Wu, B. Hu, Phys. Rev. A 35, 1404 (1987); Yu. Holovatch, Lecture Notes in Physics, 447, 224, Springer-Verlag, Heidelberg, 1996.

[28] R. Guida and J. Zinn-Justin, J. Phys. A: Math. Gen. 31, 8103 (1998).

[29] J. S. Kouvel and M. E. Fisher, Phys. Rev. 136 A1626 (1964); E. K. Riedel, F. J. Wegner, Phys. Rev. B 9, 294 (1974).

[30] M. Dudka, R. Folk, Yu. Holovatch, and D. Ivaneiko, J. Magn. Magn. Mater. 53, 243 (2003).

[31] A. Perumal, V. Srinivas, V. V. Rao, and R. A. Dunlap Phys. Rev. Lett. 91, 137202 (2003)

[32] P.C. Hohenberg and B.I Halperin, Rev. Mod. Phys., 49 435 (1977)

[33] R.Bauch, H. K. Janssen, H. Wagner, Z. Phys. B 24, 113 (1976)

[34] C. De Dominicis, Phys. Rev. B 184913 (1978).

[35] V.J. Emery, Phys. Rev. B 11, 239 (1975); S.F. Edvards and P.W. Anderson, J. Phys. F 5, 965 (1975).

[36] Our Lagrangian (9) differs from form obtained in Ref. [18] since we introduce the auxialiary field $i \tilde{\phi}$ instead of $\phi$ of Ref. [18].

[37] see e.g. J. Zinn-Justin, Quantum Field Theory and Critical Phenomena (International Series of Monographs on Physics, 92) (Oxford University Press, Oxford, 1996); H. Kleinert and V. Schulte-Frohlinde, Critical Properties of $\phi^{4}$-Theories (World Scientific, Singapore, 2001).

[38] H.K. Janssen, K. Oerding, and E. Sengespeick, J. Phys. A 28, 6073 (1995).

[39] H. Kleinert and V. Schulte-Frohlinde, Phys. Lett. B 342 284 (1995).

[40] R. Folk, Yu. Holovatch, and T. Yavors'kii, Phys. Rev. B 61, 15114 (2000).

[41] R. Schloms and V. Dohm, Europhys. Lett. 3, 413 (1987); R. Schloms and V. Dohm, Nucl. Phys. B 328639 (1989).

[42] H. J. Hardy, Divergent Series (Clarendon Press, Oxford, 1949).

[43] J.R. Chisholm, Math. Comp. 27, 841 (1973).

[44] Yu. Holovatch, V. Blavats'ka, M. Dudka, C. von Ferber, R. Folk, T. Yavors'kii, Int. J. Mod. Phys. B. 16, 4027 (2002).

[45] T. Vojta, J. Phys. A: Math. Gen. 36, 10921 (2002); R. Sknepnek and T. Vojta, Phys. Rev. B 69, 174410 (2004); B. Fendler, R. Sknepnek, T. Vojta, J. Phys. A: Math. Gen. 38, 2349 (2005).

[46] S. Wansleben and D. P. Landau, Phys. Rev. B 43, 6006 (1991)

[47] N. Ito, Physica A 192, 604 (1993).

[48] M. Kikuchi and N. Ito, J. Phys. Soc. Japan 62, 3052 (1993); M. Kikuchi, N. Ito, and Y. Okabe, preprint cond-mat/9601035

[49] P. Grassberger, Physica A, 214, 547 (1995) [Erratum: 217227

[50] N. Ito, K. Hukushima, K. Ogawa, Y. Ozeki, J. Phys. Soc. Jap. 691931 (2000)

[51] D. P. Belanger, B. Farago, V. Jaccarino, A. R. King, C. Lartigue, and F. Mezei, J. Phys. (Paris) Colloq. 49 C81229 (1988).

[52] V.V. Prudnikov,A.V. Ivanov and A.A. Fedorenko, Pis'ma Zh. Eksp. Fiz. 66793 (1997)

[53] V. V. Prudnikov, A. N. Vakilov, Sov. Phys. JETP 74, 990 (1992) [Zh. Eksp. Teor. Fiz. 101, 1853 (1992)].

[54] H.-O. Heuer, J. Phys. A: Math. Gen. 26, L341 (1993).

[55] G. Parisi, F. Ricci-Tersenghi, and J. J. Ruiz-Lorenzo, Phys. Rev. E 605198 (1999).

[56] N. Rosov, C. Hohenemser, M. Eibschütz, Phys. Rev. B 46, 3452 (1992).

[57] G.Grinstein, S.-k. Ma, and G. Mazenko, Phys. Rev. B 15 258 (1977).

[58] K. Oerding and H. K. Janssen, J. Phys. A: Math. Gen. 28, 4271 (1995).

[59] P. J. S. Watson, J. Phys. A 7, L167 (1974)

[60] A. B. Harris and T. C. Lubensky, Phys. Rev. Lett. 33 1540 (1974); D. E. Khmel'nitskii, Zh. Eksp. Teor. Fiz. 68, 1960 (1975) [Sov. Phys. JETP 41, 981 (1975)]; T. C. Lubensky, Phys. Rev. B, 11, 3573 (1975); G. Grinstein, A. Luther, Phys. Rev. B 13, 1329 (1976).

[61] H. Kleinert, J.Neu, V. Schulte-Frohlinde, K. F. Chetyrkin and S.A. Larin, Phys. Lett. B 27239 (1991).

[62] M. Dudka, Yu. Holovatch, T. Yavors'kii, J. Phys. A: Math. Gen. 37, 10727 (2004). 
[63] G. A. Baker, Jn. and P. Graves-Morris, Padé Approximants, Reading, MA: Addison-Wesley, 1981.

[64] N.V. Antonov, N.Vasil'ev, Theor. and Mat. Fiz., 6059 (1984)

[65] In general this dependence is not given by a power law but is more complicated and would have to be calculated in the non-asymptotic theory.

[66] This approximation has successfully been used in the work on diluted models [38]; 40]; 30] and in other cases: E. Frey, F. Schwabl, Phys. Rev. B 428261 (1990); I. Nasser, R. Folk, Phys. Rev. B 5215799 (1995). 